\title{
Eficiencia socio ambiental de la reacción fenton en el tratamiento de lixiviados
}

\begin{abstract}
Socio-environmental efficiency of the Fenton reaction in the treatment of leachate

José Gerardo León Chimbolema. ${ }^{1}$, Sofía Carolina Godoy Ponce. ${ }^{2}$ \& Mayra Alexandra Guevara Villegas. ${ }^{3}$
\end{abstract}

Recibido: 24-04-2020 / Revisado: 22-05-2020 / Aceptado: 24-06-2020 / Publicado: 03-07-2020

\begin{abstract}
.
DOI: https://doi.org/10.33262/cienciadigital.v4i3.1334

The research work focused on determining the conditions of socio-environmental efficiency of the Fenton process for the treatment of leachates from a landfill. The cost-benefit assessment of social and environmental variables of the treatment in winter and summer time has been carried out through the analysis of externalities and a strategic diagnosis defined by the area of direct influence and the chemical physical quality of the leachate to from the concentrations of ferrous sulfate, hydrogen peroxide, $\mathrm{pH}$, optimal dose with jug test, turbidity and color. When the sanitary landfill was cataloged as a young landfill, from the results generated there were parameters with high concentrations, so the treatment process required optimal conditions of $400 \mathrm{mg} / \mathrm{L}$ for hydrogen peroxide, $1000 \mathrm{mg} / \mathrm{L}$ for ferrous sulfate and a $\mathrm{pH}$ of 3 , values that generated an efficiency of $85 \%$ for biochemical oxygen demand, $73 \%$ for chemical oxygen demand, $91.0 \%$ turbidity and $33.3 \%$ alkalinity. These last parameters were indicators that the process represents a high socio-environmental cost - benefit for the sector under study.
\end{abstract}

Keywords: fenton, leached, socio-environmental efficiency, cost-benefit, treatment.

1 Escuela Superior Politécnica de Chimborazo, Facultad de Ciencias. Riobamba, Ecuador. gerardo.leon@espoch.edu.ec

2 Escuela Superior Politécnica de Chimborazo, Facultad de Ciencias. Riobamba, Ecuador. sofia.godoy@espoch.edu.ec

3 Escuela Superior Politécnica de Chimborazo, Facultad de Ciencias. Riobamba, Ecuador. mvillegas@espoch.edu.ec 


\section{Resumen.}

El trabajo de investigación se centró en la determinación de las condiciones de eficiencia socio ambiental del proceso Fenton para el tratamiento de lixiviados de un relleno sanitario. Se ha llevado a cabo la valoración costo-beneficio de variables sociales y ambientales del tratamiento en época de invierno y verano a través del análisis de externalidades y de un diagnóstico estratégico definido por el área de influencia directa y de la calidad físico química del lixiviado a partir de las concentraciones de sulfato ferroso, peróxido de hidrógeno, $\mathrm{pH}$, dosis óptima con prueba de jarras, turbidez y color. Al ser catalogado el relleno sanitario como un relleno joven, de los resultados generados existieron parámetros con concentraciones altas, por lo que el proceso de tratamiento requirió de condiciones óptimas de $400 \mathrm{mg} / \mathrm{L}$ para peróxido de hidrógeno, $1000 \mathrm{mg} / \mathrm{L}$ para sulfato ferroso y un $\mathrm{pH}$ de 3, valores que generaron una eficiencia de $85 \%$ para la demanda bioquímica de oxígeno, $73 \%$ para la demanda química de oxígeno, 91,0 \% de turbidez y 33,3\% de alcalinidad. Estos últimos parámetros fueron indicadores de que el proceso representa un costo - beneficio socio ambiental alto para el sector objeto de estudio.

Palabras claves: fenton, lixiviado, eficiencia socio ambiental, costo-beneficio, tratamiento.

\section{Introducción.}

El incremento de la producción de desechos sólidos industriales, agrícolas y domésticos trae consigo consecuencias de carácter socio ambientales que requieren la acción efectiva de tratamientos tecnológicos sostenibles. El lugar de disposición final de los residuos sólidos lo constituyen en la mayoría de los casos los rellenos sanitarios, lugar representativo de depósito de lixiviados y de concentración contaminante a efecto de acumulación de los residuos sólidos (Basanta, García Delgado, Cervantes Martínez, Mata Vázquez, \& Bustos Vázquez, 2007).

Mecanismos implicados para la gestión apropiada de residuos sólidos urbanos involucran su tratamiento y disposición final, para ello se efectúa una valoración de sus impactos ambientales significativos relacionados con los gases de vertedero y lixiviados generados (Pellón Arrechea, López Torres, Espinoza Llórens, \& González Díaz, 2015).

Los indicadores ambientales y económicos de los sistemas de gestión ambiental de residuos se basan el análisis y costeo del ciclo de vida. La evaluación de impactos ambientales en el análisis costo beneficio socio ambiental en América Latina actualmente considera aspectos de procesos primarios de producción y reciclaje. El análisis económico incluye costos operativos y de inversión a los costos de las externalidades ambientales, permitiendo así el análisis de los costos totales para la sociedad (Paes, y otros, 2020) 
Los lixiviados son considerados líquidos en contacto con los desechos de rellenos sanitarios y el agua. Son los líquidos percolados que se forman como resultado de la biodegradación, arrastre y filtración de la materia orgánica e inorgánica ( Sáez \& Urdaneta , 2014). El lixiviado presenta una composición variable y de elevada toxicidad, por lo que no es posible comparar resultados con otro, esto implica que debe ser caracterizado y evaluado de manera independiente ( Sáez \& Urdaneta , 2014). Los lixiviados contienen altas concentraciones de Carbono Orgánico Total (COT), Demanda Bioquímica de Oxígeno (DBO5) y Demanda química de Oxígeno (DQO), indicadores de la presencia de compuestos orgánicos y sustancias inorgánicas, elevados sólidos totales y disueltos, entre otros. Esta composición depende de la composición de los desechos y de su estabilización (Borzacconi et al., 1996).

Los tratamientos convencionales de lixiviados conllevan un alto costo de construcción, operación y mantenimiento, sin embargo, los resultados de remoción de carga contaminante en muchos casos son deficientes, por este motivo se han diseñado métodos alternativos de depuración de bajo costo y efectivos como es el caso de la tecnología fenton (Guevara, Guanoluisa, \& de la Torre, 2014). El tratamiento de los lixiviados se ha convertido así en es uno de los conflictos más relevantes y desafiantes de los rellenos sanitarios y las aguas residuales, que motiva a las organizaciones a considerar tecnologías existentes en el país, económicamente viables y de impacto significativo para la sociedad ecuatoriana (Guevara , Guanoluisa, \& de la Torre, 2014).

Los contaminantes presentes en los lixiviados varían con el tiempo, y no sería posible su tratamiento aplicando un único proceso tecnológico. Los lixiviados jóvenes presentan la relación (DBO5/DBO) mayores a 0.4, lo que implica alta aplicabilidad para tratamientos biológicos, sin embargo en lixiviados de rellenos viejos, estos tratamientos son de baja aplicabilidad (Robles, 2005). Al transcurrir el tiempo la composición va cambiando y la fracción biodegradable disminuye a una relación (DBO5/DBO) como resultado de la descomposición a sustancias estables como metano (Lau et al., 2001).

No todos los compuestos orgánicos son biodegradables, sino, sólo aquellos de bajo peso molecular. Los que no son biodegradables presentan una eficiencia limitada frente a tratamientos de tipo biológico (Yoo et al., 2001). Por su naturaleza requiere uso de procesos alternos a la biodegradación. Según (Vilar, 2015), se estima que por cada tonelada de residuos sólidos se genera $0.2 \mathrm{~m}^{3}$ de lixiviados y una vez clausurados, la generación de lixiviados puede seguir por más de 50 años.

Dentro de Procesos de Oxidación Avanzada (POA), se encuentra la oxidación Fenton como alternativa viable como pre o post tratamiento biológico (Hermosilla et al., 2009; Oller et al., 2011). Este proceso se sustenta en oxidación química por la formación de radicales libres $(* \mathrm{OH})$ que tienen alto potencial de reducción (Isarain Chávez, 2010). 
A fines del siglo XIX estudios demostraron que la solución de sales ferrosas y de peróxido de hidrógeno era capaz de oxidar compuestos orgánicos (Chevez, 1975), más tarde (Tang $\&$ Tassos, 1997) sugirieron que se forma radicales $(* \mathrm{OH})$ mediante la siguiente reacción:

$$
\mathrm{Fe}^{2+} \mathrm{aq}+\mathrm{H}_{2} \mathrm{O}_{2} \rightarrow \mathrm{Fe}^{3+}+\mathrm{OH}^{-}+\mathrm{OH}
$$

Estos radicales pueden oxidar a la materia orgánica produciendo radicales orgánicos que pueden ser oxidados por $\mathrm{Fe}^{3+}$ con la reacción que se indica (Pignatello, 1992).

$$
\begin{gathered}
R^{+}+\mathrm{Fe}^{3+} \rightarrow R^{+}+\mathrm{Fe}^{2+} \\
R^{+}+\mathrm{Fe}^{2+} \rightarrow R^{-}+\mathrm{Fe}^{3+} \\
2 R^{\cdot} \rightarrow R-R
\end{gathered}
$$

El proceso Fenton con base enestudios demuestra que es efectivo para degradar una serie de compuestos que no se degradan por métodos convencionales de tratamiento (Bigda, 1995; Primo, 2008). También existen estudios que ha reducido exitosamente la DQO de aguas residuales municipales y en el tratamiento de lixiviados.

En la actualidad existen una serie de estudios sobre la eficiencia del reactivo Fenton en la remoción de contaminantes presentes en los lixiviados de rellenos sanitarios de distintos países. La principal ventaja de tratamiento por proceso Fenton es que sus componentes son de manipulación simple, económicos y ambientalmente benignos (Kavitha \& Palanivelu, 2003).

En el relleno sanitario de la ciudad de Mérida, Yucatán, se realizaron estudios de tratamiento de lixiviado por Fenton donde el tiempo óptimo fue 20 minutos con concentración de $\mathrm{Fe}^{2+}$ de $1000 \mathrm{mg} / \mathrm{L}$ y de $600 \mathrm{mg} / \mathrm{L}$ de $\mathrm{H}_{2} \mathrm{O}_{2}$ con una remoción de $78 \%$ de DQO (Méndez et al., 2010). Similarmente se realizó el estudio fenton en la disposición final de Tuxtla Gutiérrez, Chiapas, México lográndose una eficiencia de remoción de DQO en $67 \%$, también se ha realizado el tratamiento de lixiviado del antiguo relleno sanitario "La reserva" por proceso fenton en León, Guanajuato, removiéndose la DQO en 75.3 \% (Mireles \& Páramo, 2017).

En Perú se realizó el estudio de optimización de tratamiento fenton en lixiviados de rellenos sanitarios en el que se utilizaron sistemas de jarras con agitación y la metodología de superficies de respuesta, donde se determinó que este sistema puede ser empleado como posttratamiento logrando eliminar el $42 \%$ de DQO (Medina et al., 2012, 2016; Medina Valderrama et al., 2018). En el relleno sanitario de Cantón Mejía-Quito Ecuador se realizó el estudio de lixiviado por oxidación fenton en la cual logró una eficiencia de $90 \%$ de DQO. En la ciudad de Ambato quebrada Chazinato, se realizó el estudio de tratamiento de una mezcla de agua residual doméstica con el lixiviado por el método Fenton modificado, 
obteniendo un rendimiento de DQO de 44.4 \% (Sánchez \& García Gualoto, 2018). No existen más estudios en el país relacionado al tema en referencia.

El objetivo de este trabajo fue determinar la eficiencia del proceso fenton en el tratamiento de lixiviados del relleno sanitario "Porlón" de la ciudad de Riobamba-Ecuador.

\section{Metodologia.}

El estudio socioeconómico requirió de una valoración costo-beneficio a partir de variables sociales y ambientales del tratamiento tanto en época de invierno y verano acompañado del análisis de externalidades (causa-efecto) y de un diagnóstico estratégico definido por el área de influencia directa y de la calidad físicoquímica del lixiviado a partir de las concentraciones de sulfato ferroso, peróxido de hidrógeno, $\mathrm{pH}$, dosis óptima con prueba de jarras, turbidez y color. Se sustentó en la evaluación del grado de aceptabilidad del proceso por parte de la comunidad que se determinó con base en la aplicación de estrategias sociales de campo como encuestas, entrevistas y grupos focales.

Se realizaron muestreos compuestos durante 6 horas consecutivas desde el efluente de lixiviado del relleno sanitario de Porlón en la ciudad de Riobamba. Se realizaron valoraciones socioeconómicas y a la muestra se la caracterizó a través de pruebas físico-químicas: pH, Sólidos Totales Disueltos (STD), Conductividad, Alcalinidad, Turbiedad, Color, $\mathrm{DBO}_{5}$, DQO y tensoactivos empleando las técnicas establecidas en el Standard Methods (APHAAWWA-WPCF, 2017).

Para determinar la dosis aproximada de hierro se preparó una solución patrón de sulfato ferroso utilizando $1000 \mathrm{~mL}$ de lixiviado al que colocado en el equipo de prueba de jarras a 100 rpm se le adicionó gradualmente el volumen de la disolución patrón de hierro hasta que se formaron pequeños flóculos, a éste valor se consideró como una dosis aproximada de hierro. El pH óptimo comprendió valores entre 2 y 4 a partir de ácido sulfúrico concentrado al $97 \%$ w/w. Para encontrar la dosis óptima de hierro se varió su concentración alrededor de valor de dosis aproximada, éste fue mezclado a $100 \mathrm{rpm}$ durante 1 minuto, con una velocidad modificada a $30 \mathrm{rpm}$ por 12 minutos para finalmente permanecer en reposo durante 30 minutos más (Méndez, García, Castillo, \& Sauri, 2010).

La concentración óptima de peróxido de hidrógeno se determinó variándola desde $200 \mathrm{mg} / \mathrm{L}$ hasta $800 \mathrm{mg} / \mathrm{L}$ y mezclándola con la dosis de hierro. La mezcla fue agitada a $100 \mathrm{rpm}$ durante 1 minuto, la velocidad de agitación se modificó a $30 \mathrm{rpm}$ por el lapso de 12 minutos y un tiempo de en reposo de 30 minutos. A partir del mencionado proceso se determinó la turbidez, color y las condiciones óptimas del reactivo fenton, variando el pH a concentración óptima y parámetros extremos. 
Para determinar la eficiencia del proceso se realizaron pruebas con los parámetros fenton obtenidos por triplicado con su respectiva caracterización antes y después del tratamiento.

\section{Resultados.}

El método empleado trae consigo ventajas comparativas en relación al componente económico: ahorro respecto a procesos convencionales, relacionadas a un diseño simple, componente ambiental: proceso que incentiva el máximo aprovechamiento de los recursos naturales y que no genera compuestos estables residuales perjudiciales para la comunidad, componente social: proyecto de innovación, dentro del marco de la investigación se emplearon tecnologías y metodologías relativamente nuevas que exploraron la capacitad local con el uso de materiales de trabajo de fácil acceso y manejo en el mercado.

En la tabla 1, se presenta resultados de la caracterización de lixiviado en 2 épocas climáticas (invierno y verano) con los parámetros más importantes.

En la tabla 2, se indica los valores de tratamiento realizado y obtenidos como turbidez y remoción de color con sus distintas combinaciones de acuerdo a la metodología planteada.

En la tabla 3, muestra el comportamiento del pH a la variación del reactivo fenton, la tabla 4 presenta la eficiencia del proceso bajo condiciones óptimas encontradas en el laboratorio.

De acuerdo a los resultados de la tabla 1, el pH se encontró en medio básico y no existió variabilidad en las épocas del año, estudios realizados en Estados Unidos y Noruega, reportaron valores de $\mathrm{pH}$ entre 5,4 y 7 (Słomczyńska \& Słomczyński, 2004), el valor de pH obtenido se debió al tipo de cobertura empleada en el relleno sanitario (Méndez et al., 2010).

La $\mathrm{DBO}_{5}$ representó valores altos tanto en invierno como en verano, $16200 \mathrm{mg} / \mathrm{L}$ y 27000 $\mathrm{mg} / \mathrm{L}$ respectivamente lo que constituyó en un indicador de alto contenido de compuestos orgánicos en la fase de acidogénesis entre 2 y 15 años de operación.

Cuando el tiempo de operación es mayor a 15 años las concentraciones de éstos parámetros fueron bajos (Słomczyńska \& Słomczyński, 2004)(Méndez et al., 2004), y al comparar los resultados entre las dos épocas del año se definieron diferencias marcadas con base en una inapropiada impermeabilización del relleno sanitario, provocando el aumento de aguas lluvia durante el escurrimiento del lixiviado con efecto de dilución (Espinosa et al., 2010).

La relación $\mathrm{DBO}_{5} / \mathrm{DQO}$, indicó que la muestra fue muy biodegradable. 
Tabla 1. Caracterización de lixiviados generados en el relleno sanitario Porlón de la ciudad de Riobamba

\begin{tabular}{lccccccccc}
\hline \multirow{2}{*}{ Parámetros } & Unidad & \multicolumn{4}{c}{ Invierno } & \multicolumn{4}{c}{ Verano } \\
\cline { 3 - 10 } & & Mínimo & Promedio & Máximo & Desviación & Mínimo & Promedio & Máximo & Desviación \\
\hline pH & --- & 8,2 & 8,3 & 8,4 & 0,0817 & 8,2 & 8,3 & 8,4 & 0,0846 \\
STD & $\mathrm{mg} / \mathrm{L}$ & 5148 & 5150 & 5151 & 1,2645 & 8099 & 8100 & 8104 & 2,1723 \\
Conductividad & $\mathrm{ms} / \mathrm{cm}$ & 9.1 & 9.3 & 9.5 & 0,1643 & 11,8 & 12,4 & 13,4 & 0,6735 \\
Alcalinidad & $\mathrm{mg} / \mathrm{L}$ & 545 & 550 & 553 & 3,3043 & 298 & 300 & 308 & 4,3541 \\
Turbidez & $\mathrm{NTU}$ & 1198 & 1200 & 1204 & 2,4837 & 1815 & 1820 & 1825 & 2,7632 \\
Color & Pt-Co & 24048 & 24050 & 24053 & 2,0745 & 31097 & 31100 & 31103 & 2,4692 \\
DBO5 & $\mathrm{mg} / \mathrm{L}$ & 16198 & 16200 & 16210 & 3,986 & 26998 & 27000 & 27002 & 1,7632 \\
DQO & $\mathrm{mg} / \mathrm{L}$ & 20499 & 20500 & 20510 & 3,8746 & 26095 & 26100 & 26106 & 4,5823 \\
Tensoactivos & $\mathrm{mg} / \mathrm{L}$ & --- & --- & --- & --- & 2,5 & 2,7 & 2,9 & 0,0173 \\
\hline
\end{tabular}

Fuente: Elaboración propia.

El color fue uno de los parámetros característico de los lixiviados que en verano resultó ser mayor que en invierno, ratificando el efecto de dilución por precipitación, valores de color menor a $7000 \mathrm{mg} / \mathrm{L}$ en época de lluvia corresponden a un lixiviado viejo (Espinosa et al., 2010). La cantidad de dosis óptima para hierro correspondió a la concentración de $1000 \mathrm{mg} / \mathrm{L}$ en que se determinó una menor cantidad de turbidez y una alta remoción de color $(87,0 \%)$, tal como se indica en las figuras 1 y 2 . Al variar la concentración de peróxido de hidrógeno y concentración constante de sulfato ferroso, se observó que a $400 \mathrm{mg} / \mathrm{L}$ de $\mathrm{H}_{2} \mathrm{O}_{2}$ menos valor de turbidez $(90,8 \%)$ y color $(86,7 \%)$ presentan las muestras, resultados que se visualizan en las figuras 3 y 4.

Figura 1. Dosis óptima de Fe2+ al mantener peróxido constante

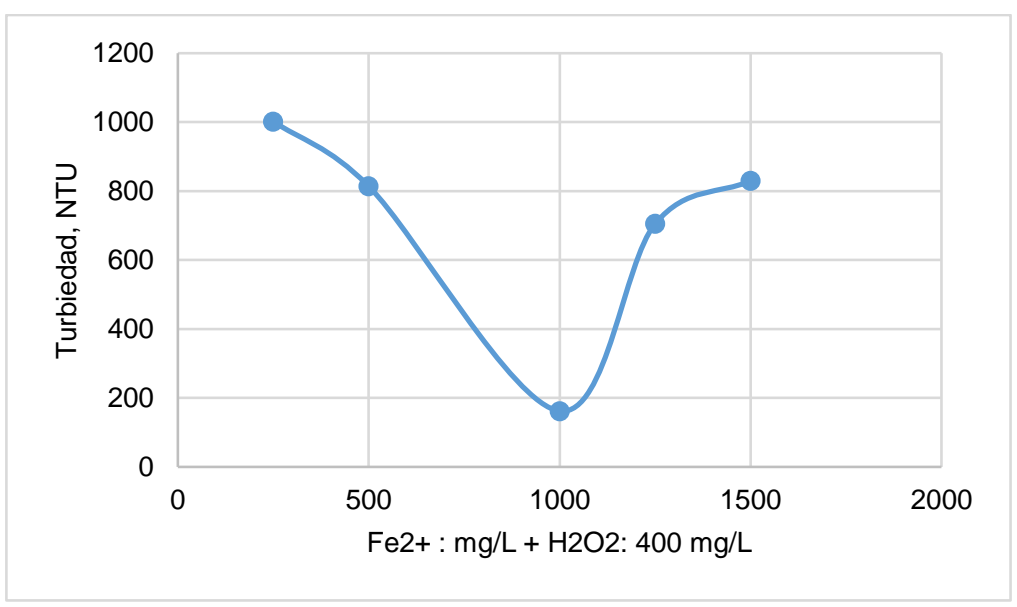

Fuente: Elaboración propia. 
Tabla 2. Porcentaje de remoción de turbiedad y color en el tratamiento Fenton

\begin{tabular}{|c|c|c|c|c|c|c|}
\hline \multirow{2}{*}{$\begin{array}{c}\text { Dosis } \\
\mathrm{H}_{2} \mathrm{O}_{2} \\
(\mathrm{mg} / \mathrm{L})\end{array}$} & \multirow{2}{*}{$\begin{array}{c}\text { Dosis Fe }^{2+} \\
(\mathrm{mg} / \mathrm{L})\end{array}$} & \multirow[b]{2}{*}{ pH } & \multicolumn{2}{|c|}{ Turbiedad } & \multicolumn{2}{|c|}{ Color } \\
\hline & & & NTU & $\begin{array}{c}\text { Remoción } \\
(\%)\end{array}$ & Pt-Co & $\begin{array}{c}\text { Remoción } \\
(\%)\end{array}$ \\
\hline 400 & 250 & 2 & 1721 & 5,0 & 25346 & 18,0 \\
\hline 400 & 500 & 2 & 1667 & 8,0 & 24728 & 20,0 \\
\hline 400 & 1000 & 2 & 1540 & 15,0 & 23800 & 23,0 \\
\hline 400 & 1250 & 2 & 1644 & 9,3 & 23400 & 24,3 \\
\hline 400 & 250 & 3 & 1002 & 44,5 & 17456 & 42,4 \\
\hline 400 & 500 & 3 & 814 & 54,9 & 12032 & 60,3 \\
\hline 400 & 1000 & 3 & 162 & 91,0 & 3940 & 87,0 \\
\hline 400 & 1250 & 3 & 705 & 60,9 & 10395 & 65,7 \\
\hline $\begin{array}{l}400 \\
400\end{array}$ & $\begin{array}{c}1500 \\
250\end{array}$ & $\begin{array}{l}3 \\
4\end{array}$ & $\begin{array}{c}830 \\
1366\end{array}$ & $\begin{array}{l}78,7 \\
25,0\end{array}$ & $\begin{array}{l}13547 \\
19127\end{array}$ & $\begin{array}{l}55,3 \\
38,0\end{array}$ \\
\hline 400 & 500 & 4 & 1129 & 38,0 & 16044 & 48,0 \\
\hline 400 & 1000 & 4 & 1020 & 44,0 & 14500 & 53,0 \\
\hline 400 & 1250 & 4 & 1184 & 35,0 & 16654 & 46,0 \\
\hline 400 & 1500 & 4 & 1311 & 28,0 & 19750 & 36,0 \\
\hline 200 & 1000 & 2 & 1641 & 4,0 & 25278 & 18,1 \\
\hline 400 & 1000 & 2 & 1538 & 10,0 & 23797 & 22,8 \\
\hline 600 & 1000 & 2 & 1572 & 8,0 & 23704 & 23,2 \\
\hline 800 & 1000 & 2 & 1624 & 5,0 & 23426 & 24,1 \\
\hline 200 & 1000 & 3 & 609 & 65,5 & 15238 & 48,3 \\
\hline 400 & 1000 & 3 & 161 & 90,8 & 3920 & 86,7 \\
\hline 600 & 1000 & 3 & 448 & 74,6 & 11878 & 59,7 \\
\hline 800 & 1000 & 3 & 557 & 68,4 & 16329 & 44,6 \\
\hline 200 & 1000 & 4 & 1315 & 27,0 & 18923 & 38,4 \\
\hline 400 & 1000 & 4 & 1012 & 43,8 & 14510 & 52,8 \\
\hline 600 & 1000 & 4 & 1225 & 32,0 & 15514 & 49,5 \\
\hline 800 & 1000 & 4 & 1441 & 20,0 & 18770 & 38,9 \\
\hline
\end{tabular}

Fuente: Elaboración propia.

Según los datos de la tabla 3 y figura 4 se observó que a $\mathrm{pH}=3$ se mantiene la mejor eficiencia de remoción de turbidez y color, lo que indica que el $\mathrm{pH}$ no varía al modificar la concentración del reactivo fenton, también se observó que la mezcla de $600 \mathrm{mg} / \mathrm{L}$ de peróxido y $1250 \mathrm{mg} / \mathrm{L}$ de hierro da buenos resultados. Sin embargo (Rivas et al., 2004), reporta que la condición óptima de $\mathrm{pH}$ es de 3.5, lo que denota la gran dependencia de la composición de lixiviado en estudio. La variación de $\mathrm{pH}$ afecta en la velocidad de reacción (Rubio et al., 2014) 
Figura 2. Eficiencia en remoción de color con variación de $\mathrm{Fe}^{2+}$

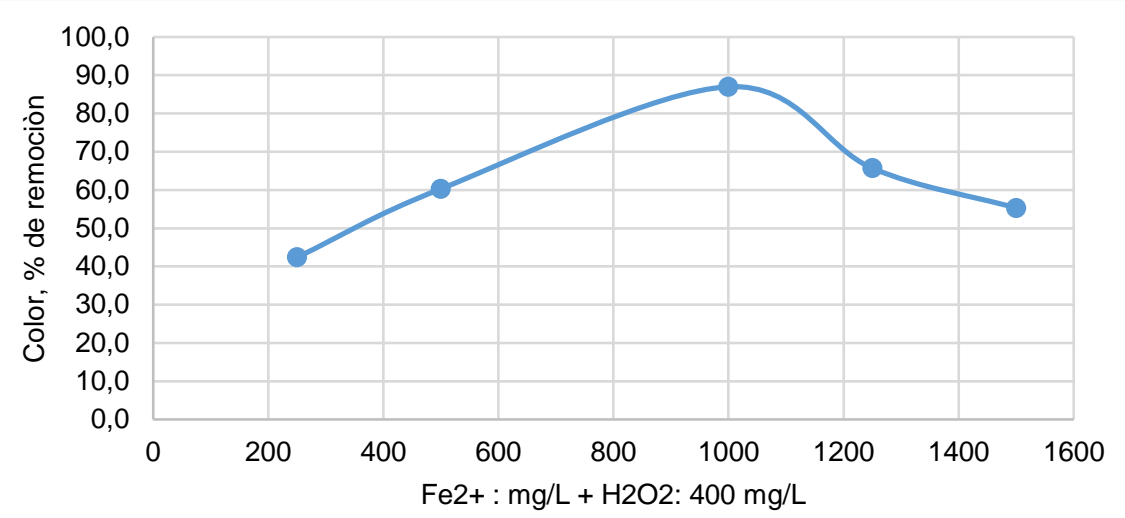

Fuente: Elaboración propia.

Figura 3. Dosis óptima de $\mathrm{H} 2 \mathrm{O} 2$ al mantener hierro constante

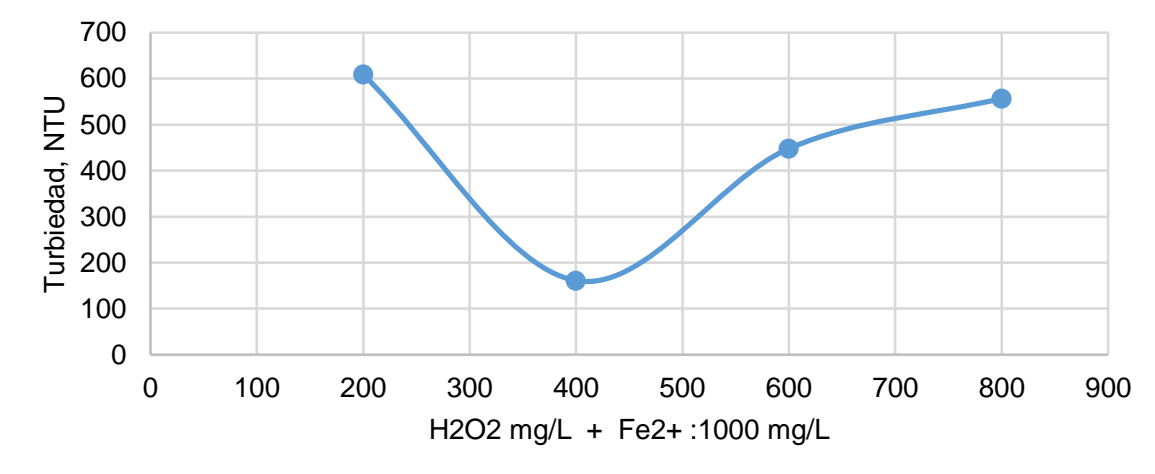

Fuente: Elaboración propia.

Figura 4. Eficiencia en remoción de color con variación de $\mathrm{H} 2 \mathrm{O} 2$

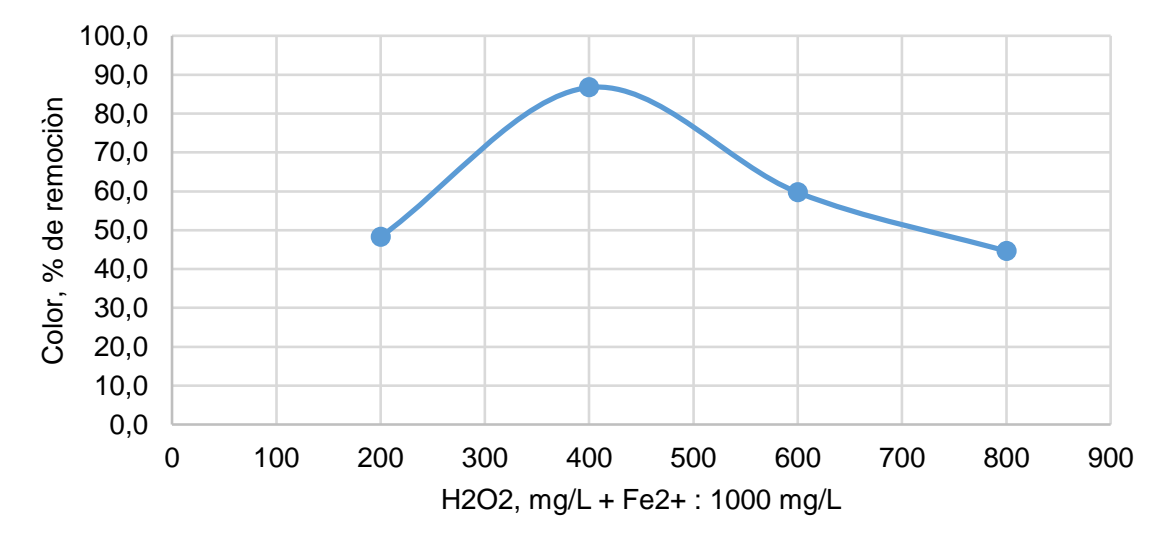

Fuente: Elaboración propia. 
Tabla 3. Análisis de $\mathrm{pH}$ en condiciones distintas del reactivo fenton

\begin{tabular}{ccccccc}
\hline $\mathbf{p H}$ & $\begin{array}{c}\mathbf{H}_{2} \mathbf{O} 2 \\
\mathbf{m g} / \mathbf{L}\end{array}$ & $\begin{array}{c}\mathbf{F e}^{2+} \\
\mathbf{m g} / \mathbf{L}\end{array}$ & $\begin{array}{c}\text { Turbiedad, } \\
\text { NTU }\end{array}$ & $\begin{array}{c}\text { \% Removido } \\
\text { Turbiedad }\end{array}$ & $\begin{array}{c}\text { Color } \\
\text { Pt-Co }\end{array}$ & $\begin{array}{c}\text { \% Removido } \\
\text { color }\end{array}$ \\
\hline 2 & 600 & 1250 & 1644 & 10,1 & 23400 & 25,1 \\
3 & 600 & 1250 & 170 & 90,6 & 4120 & 85,9 \\
4 & 600 & 1250 & 1200 & 67,2 & 10800 & 65,1 \\
2 & 400 & 1000 & 1540 & 14,2 & 23800 & 23,1 \\
3 & 400 & 1000 & 162 & 90,9 & 3940 & 86,8 \\
4 & 400 & 1000 & 1020 & 43,7 & 14500 & 52,7 \\
\hline
\end{tabular}

Fuente: Elaboración propia.

Figura 5. Variación de $\mathrm{pH}$ a dos reactivos Fenton

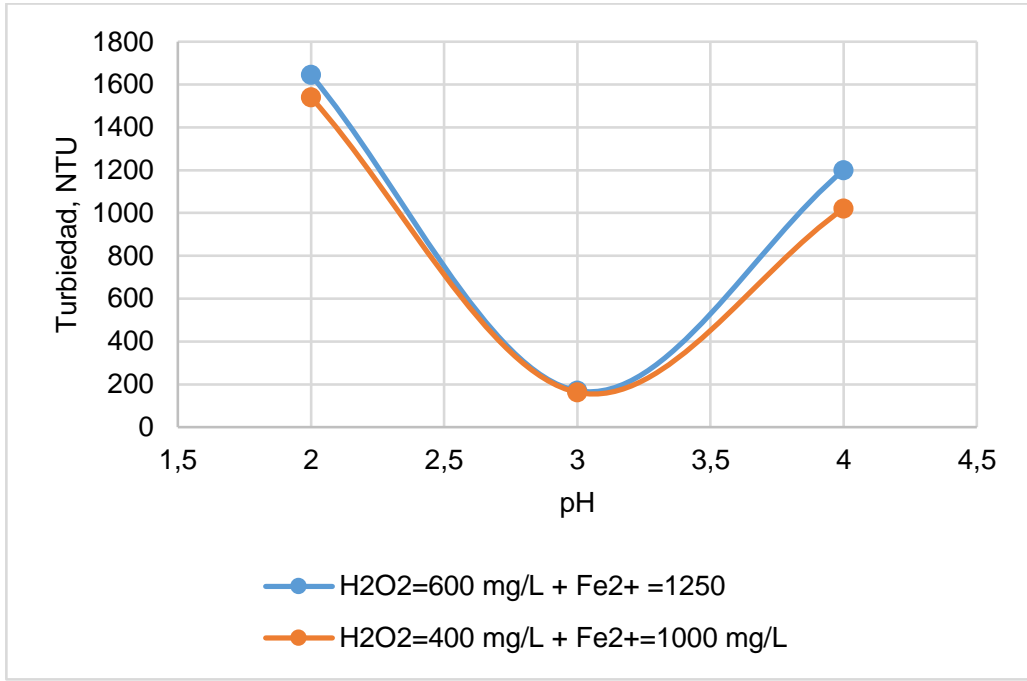

Fuente: Elaboración propia.

Tabla 4. Eficiencia del tratamiento Fenton en eliminación de contaminantes de lixiviados

\begin{tabular}{lccccccc}
\hline & & \multicolumn{3}{c}{ Verano } & \multicolumn{3}{c}{ Invierno } \\
\cline { 3 - 8 } Parámetros & Unidad & inicial & Final & $\begin{array}{c}\text { Eficiencia, } \\
\%\end{array}$ & Inicial & Final & $\begin{array}{c}\text { Eficiencia, } \\
\%\end{array}$ \\
\hline $\mathrm{pH}$ & --- & 8.5 & 3 & --- & 8.3 & 3 & --- \\
STD & $\mathrm{g} / \mathrm{L}$ & 8.1 & 2 & 75,3 & 5150 & 1180 & 77,1 \\
Conductividad & $\mathrm{ms} / \mathrm{cm}$ & 14.3 & 4 & 72,0 & 9.3 & 2.6 & 72,0 \\
Alcalinidad & $\mathrm{mg} / \mathrm{L}$ & 300 & 200 & 33,3 & 550 & 350 & 36,4 \\
Turbidez & NTU & 1820 & 162 & 91,0 & 1200 & 150 & 87,5 \\
Color & pt Co & 31100 & 3940 & 87,3 & 24050 & 3700 & 84,6
\end{tabular}


ISSN: 2602-8085

\begin{tabular}{lccccccc}
$\mathrm{DBO}_{5}$ & $\mathrm{mg} / \mathrm{L}$ & 27000 & 4050 & 85,0 & 16200 & 2200 & 86,4 \\
$\mathrm{DQO}$ & $\mathrm{mg} / \mathrm{L}$ & 26100 & 7050 & 73,0 & 20500 & 5350 & 73,9 \\
Tensoactivos & $\mathrm{mg} / \mathrm{L}$ & 2.73 & 1.35 & 50,5 & --- & --- & --- \\
\hline
\end{tabular}

Fuente: Elaboración propia.

Según los datos de la tabla 4, el parámetro con mayor eficiencia en la remoción fue la turbidez con $91.0 \%$ en verano y $87.5 \%$ en invierno, el color en $87.3 \%$ en verano y $84.6 \%$ en invierno, la DBO sufrió una reducción de $85 \%$ en época de verano y $86.4 \%$ en invierno, los sólidos totales disueltos también se redujeron en $75.3 \%$. La DQO se ha reducido en $73 \%$. La alcalinidad fue el componente que menor reducción ha sufrido en las dos épocas de análisis $33.3 \%$ a $36.4 \%$. Estudios realizados en Huancayo-Perú, indicaron que la eficiencia a pH con un valor de 3.9, sulfato ferroso con 1645,55 mg/L, peróxido de hidrógeno de 1096.55 $\mathrm{mg} / \mathrm{L}$ obtuvo una eficiencia de $42 \%$ en términos de DQO en las dos épocas de estudio (Medina et al., 2012; Medina Valderrama et al., 2018). Estudios realizados en Guanajuato, reportaron eficiencia de $96,88 \%$ en turbidez y $81.38 \%$ en turbidez para una reacción fenton de $1250 \mathrm{mg} / \mathrm{L}$ de sulfato ferroso y $1000 \mathrm{mg} / \mathrm{L}$ de peróxido de hidrógeno a $\mathrm{pH}=4$ (Huichapa et al., 2003).

\section{Conclusiones.}

- La eficiencia del proceso fenton para tratamiento de lixiviados se derivó de un adecuado análisis de las condiciones de costo-beneficio social, económico y ambiental que a través de estrategias metodológicas de trabajo en campo definieron las condiciones óptimas para alcanzar la condición de bienestar en la comunidad del área de influencia directa. El tratamiento de lixiviados a partir del proceso fenton abarató costos de producción, fomentó el uso de materiales primarios y renovables resaltando las oportunidades de desarrollo local.

- De acuerdo a los resultados finales se obtuvieron las condiciones óptimas del proceso fenton $400 \mathrm{mg} / \mathrm{L}$ de peróxido de hidrógeno con $1000 \mathrm{mg} / \mathrm{L}$ de sulfato de hierro a $\mathrm{pH}$ de 3 , donde el porcentaje de remoción que se ha logrado para la Demanda Bioquímica de Oxígeno fue de 85,0 \% en verano y 86,4 \% en invierno, la Demanda Química de Oxígeno de $73,0 \%$ en invierno y $73,9 \%$ en verano, la turbidez en $91,0 \%$ en invierno y $87,5 \%$ en verano, la disminución de color en $87,3 \%$ para verano y $84,6 \%$ en invierno. Se ha logrado disminuir en $72,0 \%$ la conductividad y en menor porcentaje respecto a la alcalinidad en $33,3 \%$ en verano y $36,4 \%$ en invierno.

- A pesar de que la eficiencia del tratamiento fue alta, no fueron suficientes sus características para descargar el lixiviado a un cuerpo hídrico, resaltando el hecho de que se requieren complementariamente otros tipos de tratamientos para el cumplimiento de la norma ambiental vigente. 


\section{Referencias bibliográficas.}

APHA-AWWA-WPCF. (2017). Standards Methods for the Examination of Water and Wastewater.

Bigda, R. J. (1995). Consider Fenton chemistry for waste-water treatment. Chemical Engineering Progress, 0-62.

Borzacconi, L., López, I., \& Anido, C. (1996). Metodología. Critical Review, March.

Chevez, W. (1975). Fenton's Reagents revisited. Acc. Chem. Res., 8, 125-131 FG - 0.

Espinosa, C., López, M., Pellón, A., Robert, M., Diaz, S., González, A., Rodríguez, N., \& Alejandro, F. (2010). Análisis del comportamiento de los lixiviados generados en un vertedero de residuos sólidos municipales de la ciudad de la Habana. Revista Internacional de Contaminación Ambiental, 26(4), 313-325.

Hermosilla, D., Cortijo, M., \& Huang, C. P. (2009). Optimizing the treatment of landfill leachate by conventional Fenton and photo-Fenton processes. The Science of the Total Environment, 407(11),

3473-3481. http://www.ncbi.nlm.nih.gov/sites/entrez?Db=pubmed\&DbFrom=pubmed\&Cmd=L ink $\&$ LinkName $=$ pubmed_pubmed $\&$ LinkReadableName $=$ Related Articles\&IdsFromResult=19278717\&ordinalpos=3\&itool=EntrezSystem2.PEntrez. Pubmed.Pubmed_ResultsPanel.Pubmed_RVDocSum

Huichapa, A., Cuevas, G., \& Arodí, M. (2003). Tratamiento De Lixiviados Generados En Un Sitio De Disposicion Disposicion Final De Rsu Mediante Los Procesos Fenton Y Fotofenton. Semarnat.

Isarain Chávez, E. (2010). Mineralization of the drug $\beta$-blocker atenolol by electro-Fenton and photoelectro-Fenton using an air-diffusion cathode for. Applied Environmental, 361-369.

Kavitha, V., \& Palanivelu, K. (2003). Degradation of 2-chlorophenol by Fenton and photoFenton processes--a comparative study. J Environ Sci Health A Tox Hazard Subst Environ Eng, 2115-1231 FG - 0.

Lau, I. W. C., Wang, P., \& Fang, H. H. P. (2001). Organic removal of anaerobically treated leachate by Fenton Coagulation. Journal of Environmental Engineering, 127(7), 666669. https://doi.org/10.1061/(ASCE)0733-9372(2001)127:7(666)

Medina, C., Montero, E., \& Cruz, L. (2012). Optimización del proceso fenton en el tratamiento de rellenos sanitarios. Universitas Psychologica, 11(1), 197-206.

Medina, C., Montero, E., \& Cruz, L. (2016). Optimización Del Proceso Fenton En El 
Tratamiento De Lixiviados De Rellenos Sanitarios. Revista de La Sociedad Química Del Perú, 82, 454-466.

Medina Valderrama, C. J., Montero del Águila, E. M., \& Cruz Pio, L. E. (2018). Optimización del proceso Fenton en el tratamiento de lixiviados de rellenos sanitarios. Revista de La Sociedad Química Del Perú, 84(3), 454-466.

Méndez, R., Castillo, E., Sauri, M., Quintal, C., Giacoman, G., \& Jiménes, B. (2004). Tratamiento fisicoquímico de los lixiviados de un relleno sanitario. Ingeniería, 8(2), $155-163$.

Méndez, R., García, R., Castillo, E., \& Sauri, M. (2010). Tratamiento de lixiviados por oxidación Fenton. Ingenieria e Investigación, 30(1), 80-85.

Mireles, H., \& Páramo, J. (2017). Tratamiento del lixiviado del antiguo relleno sanitario La Reserva mediante procesos Fenton y fisicoquímico. Revista de Ingeniería Tecnológica, 1-12.

Oller, I., Malato, S., \& Sánchez-Pérez, J. A. (2011). Combination of Advanced Oxidation Processes and biological treatments for wastewater decontamination--a review. The Science of the Total Environment, 409(20), 4141-4166. http://www.ncbi.nlm.nih.gov/sites/entrez?Db=pubmed $\&$ DbFrom=pubmed $\& C m d=L$ ink\&LinkName=pubmed_pubmed\&LinkReadableName=Related Articles\&IdsFromResult=20956012\&ordinalpos=3\&itool=EntrezSystem2.PEntrez. Pubmed.Pubmed_ResultsPanel.Pubmed_RVDocSum

Pignatello, J. J. (1992). Dark and photoassisted Fe3+ catalyzed degradation of chlorophenox herbicides by hydrogen peroxide. Environ Sci Technol, 26, 944-951.

Primo, O. (2008). Mejoras en el tratamiento de lixiviados de vertedero de RSU mediante procesos de oxidacion avanzada. 109.

Rivas, F. J., Beltrán, F., Carvalho, F., Acedo, B., \& Gimeno, O. (2004). Stabilized leachates: Sequential coagulation-flocculation + chemical oxidation process. Journal of Hazardous Materials, 116(1-2), 95-102. https://doi.org/10.1016/j.jhazmat.2004.07.022

Robles, F. M. (2005). Generación de biogás y lixiviados en los rellenos sanitarios.

Rubio, A., Chica, E., \& Peñuela, G. (2014). Application of Fenton process for treating petrochemical wastewater. Scielo, 16(2), 211-223.

Sánchez, R. G., \& García Gualoto, K. J. (2018). Tratamiento de aguas residuales con cargas industriales con oxidación avanzada en sistemas convencionales. La Granja, 27(1), 103-111. https://doi.org/10.17163/lgr.n27.2018.08 
Słomczyńska, B., \& Słomczyński, T. (2004). Physico-chemical and toxicological characteristics of leachates from MSW landfills. In Polish Journal of Environmental Studies (Vol. 13, Issue 6, pp. 627-637). http://www.pjoes.com/Physico-Chemicaland-Toxicological-Characteristics-r-nof-Leachates-from-MSWLandfills, 87707,0,2.html

Tang, W., \& Tassos, S. (1997). Oxidation kinetics and mechanism of trihalomethanes by Fenton's Reagent>. Water Research. 31, 0-11.

Tatsi, A. A., Zouboulis, A. I., Matis, K. A., \& Samaras, P. (2003). Coagulation-flocculation pretreatment of sanitary landfill leachates. Chemosphere, 53(7), 737-744. http://www.ncbi.nlm.nih.gov/sites/entrez?Db=pubmed \&DbFrom=pubmed $\&$ Cmd=L ink \&LinkName=pubmed_pubmed $\&$ LinkReadableName $=$ Related Articles\&IdsFromResult=13129513\&ordinalpos=3\&itool=EntrezSystem2.PEntrez. Pubmed.Pubmed_ResultsPanel.Pubmed_RVDocSum

Vilar, A. (2015). Evaluación del tratamiento integral del lixiviado de vertedero de residuos sólidos urbanos. Universidade Da Coruña., 228.

Yoo, H. C., Cho, S. H., \& Ko, S. O. (2001). Modification of coagulation and Fenton oxidation processes for cost-effective leachate treatment. Journal of Environmental Science and Health. Part A, Toxic/Hazardous Substances \& Environmental Engineering, 36(1), $39-48$.

http://www.ncbi.nlm.nih.gov/sites/entrez?Db=pubmed\&DbFrom=pubmed $\&$ Cmd=L ink \&LinkName=pubmed_pubmed $\&$ LinkReadableName $=$ Related Articles\&IdsFromResult=11381784\&ordinalpos=3\&itool=EntrezSystem2.PEntrez. Pubmed.Pubmed_ResultsPanel.Pubmed_RVDocSum

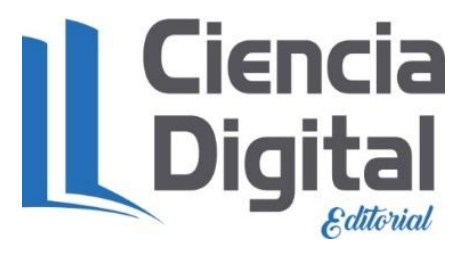


PARA CITAR EL ARTÍCULO INDEXADO.

León Chimbolema, J. G., Godoy Ponce, S. C., \& Guevara Villegas, M. A. (2020). Eficiencia socio ambiental de la reacción fenton en el tratamiento de lixiviados. Ciencia Digital, 4(3), 271-285. https://doi.org/10.33262/cienciadigital.v4i3.1334

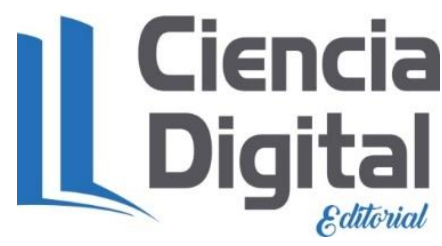

El artículo que se publica es de exclusiva responsabilidad de los autores y no necesariamente reflejan el pensamiento de la Revista Ciencia Digital.

El artículo queda en propiedad de la revista y, por tanto, su publicación parcial y/o total en otro medio tiene que ser autorizado por el director de la Revista Ciencia Digital.
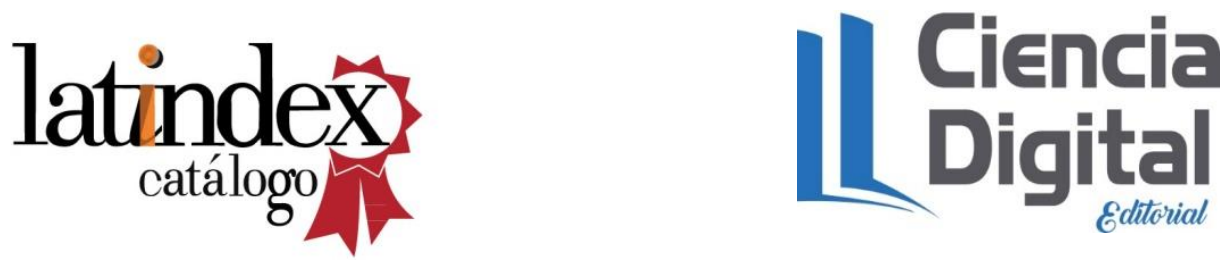